\title{
Construct domain analysis of patient health- related quality of life: physical and mental trajectory profiles following open versus endovascular repair of abdominal aortic aneurysm
}

\author{
This article was published in the following Dove Press journal: \\ Patient Related Outcome Measures \\ 27 December 2012 \\ Number of times this article has been viewed
}

\section{Nicolas J Mouawad \\ Stefan W Leichtle \\ Jeffrey $V$ Manchio \\ Richard M Lampman \\ Brian G Halloran \\ Walter MWhitehouse Jr}

Michigan Heart and Vascular Institute, Saint Joseph Mercy Health System, Ann Arbor, MI, USA
Correspondence: Nicolas | Mouawad Michigan Heart and Vascular Institute, Saint Joseph Mercy Health System,

Suite R-2 III, PO Box 995,

Ann Arbor, MI 48106, USA

$\mathrm{Tel}+1734272$ I315

Fax +I 734 7I22054

Email nmouawad@gmail.com
Purpose: Many clinical trials comparing the outcomes of open surgical repair (OSR) versus endovascular aneurysm repair (EVAR) for abdominal aortic aneurysms (AAAs) have been conducted, with varying results. Surprisingly, few outcomes studies have closely examined perceived physical and mental health-related quality of life (HRQOL) factors through a validated survey tool. The purpose of this prospective observational study was to describe the trajectory of HRQOL measures, from baseline to 1 year after surgery, in patients undergoing OSR or EVAR for AAA, and to explore for differences in physical and mental composite scores and their construct domains (subscales) using the Medical Outcomes Study 36-Item Short-Form Health Survey $\left(\mathrm{SF}-36^{\circledR}\right)$ tool.

Patients and methods: Over an 18-month period, a small sample of patients undergoing elective AAA repair in a community hospital setting were prospectively enrolled. Fifteen patients undergoing OSR and twenty patients undergoing EVAR were studied. Physical and mental HRQOL parameters were assessed using the SF-36.

Results: No significant differences in demographic and clinical variables were found between the OSR and EVAR groups. In the multivariable linear models with repeated measures, both groups showed a significant decline in physical health composite scores 30 days after the surgical procedure $(P<0.01)$. However, although the OSR group showed a statistically significant decline in three of the four physical health domains, the EVAR group declined in only one physical health domain. Only the OSR group showed a significant decline in three of the four mental health domains at 30 days; however, the decline of these domains was not reflected in the group's mental health composite scores. By 90 days after surgery, both groups were not significantly different from their baseline in physical or mental health composite scores, or in any of their respective physical health domains.

Conclusion: In this small sample of patients undergoing AAA repair, EVAR resulted in less physical and emotional decline than OSR in the early postoperative period. However, patients in both groups may return to near baseline status at 90 days.

Keywords: AAA, endovascular aneurysm repair (EVAR), open surgical repair (OSR), Medical Outcomes Study 36-Item Short-Form Health Survey (SF-36 ${ }^{\circledR}$ ), health related quality of life (HRQ)

\section{Introduction}

The prevalence of diagnosed abdominal aortic aneurysm (AAA) in the United States, determined by various methods, is estimated to be between $19 \%$ and $24 \%$ for women and between $66 \%$ and $81 \%$ for men. ${ }^{1}$ Approximately 15,000 deaths per year are caused 
by ruptured AAAs and dissections; the number may be as high as 30,000 if undiagnosed cases resulting in sudden death are considered. ${ }^{2,3}$

Conventional open surgical repair (OSR) for AAA was first performed in 1951. With the evolution of surgical techniques and aggressive critical care, the perioperative mortality rate ranges from $2 \%$ to $6 \%{ }^{4-7}$ In 1991 , Parodi et al ${ }^{8}$ introduced endovascular abdominal aortic aneurysm repair (EVAR), a minimally invasive intervention. This technique has gained wide acceptance in patients with favorable anatomic features and/or in those in whom the surgical stress of the traditional open repair is prohibitive. ${ }^{8}$

Many clinical trials have been conducted comparing OSR and EVAR outcomes of morbidity and mortality, with varying results. ${ }^{9}$ Surprisingly, few outcomes studies have closely examined perceived physical and mental healthrelated quality of life (HRQOL) factors through a validated survey tool following OSR and EVAR. ${ }^{10-15}$ When discussing the advantages of one procedure over the other with prospective patients, the impact on postoperative HRQOL may be important. As such, the present authors became interested in better understanding the association of each surgical procedure with physical and mental HRQOL, as well as aiming to better delineate how these surgical procedures for AAA repair affect the time course of recovery with respect to perceived HRQOL.

The purpose of this prospective observational study was to describe the trajectory of HRQOL measures from baseline to 1 year after AAA repair in patients undergoing OSR or EVAR, and to explore for differences in physical and mental health composite scores and their construct domains (subscales) using the Medical Outcomes Study 36-Item Short-Form Health Survey $\left(\mathrm{SF}-36^{\circledR}\right)$ tool. ${ }^{16-18}$

\section{Material and methods General study design}

Over an 18-month period, 35 consecutive patients undergoing elective AAA repair in a community hospital setting were prospectively enrolled in this study. The Institutional Review Board at Saint Joseph Mercy Health System approved the investigation, and each participant gave fully informed written consent before enrollment.

Physical and mental HRQOL parameters were assessed using the SF-36 (Medical Outcomes Trust, Inc, Waltham, MA, USA). ${ }^{16-18}$ This survey is a validated tool that generically assesses two major dimensions providing two composite scores: (1) physical health composite score, constructed from the four domains of physical function $(\mathrm{PF})$, role physical (ie, role limitations due to physical function, RP), bodily pain (BP), and general health, and (2) mental health composite score, constructed from the four domains of vitality (VT), social function (SF), role emotional (ie, role limitations due to emotional health, RE), and mental health (MH) (Table 1).

Patients completed the SF-36 surveys at their preadmission visit and then postoperatively at 30, 90, and 365 days. During the follow-up period, the questionnaire was mailed to each patient's home approximately 1 week prior to when it was required to be completed, and this was sent with a request to have the questionnaire returned within 1 week via a prepaid postage return envelope. A research nurse telephoned each participant to assess his or her progress with completing the questionnaire and to ensure the form's timely return.

Five board-certified vascular surgeons performed the AAA surgical repairs. The surgical procedure chosen was at the discretion of the surgeon and was based on patient suitability, anatomic favorability, associated comorbidities, and patient acceptance.

\section{Statistical methods}

Demographic and baseline variables were summarized using the mean plus or minus standard deviation or the percentage with $95 \%$ confidence interval, as indicated. Univariate associations of demographic and clinical variables with surgical procedure were examined using the Wilcoxon rank sum test for continuous variables and chi-square or Fisher's exact test for categorical variables.

Analyses were performed using $\mathrm{SAS}^{\circledR}$ software (v 9.1; SAS Institute Inc, Cary, NC, USA). Multivariable linear

Table I Brief explanation of physical and mental health domains ${ }^{a}$

\begin{tabular}{|c|c|}
\hline Domain & Brief definition \\
\hline \multicolumn{2}{|l|}{ Physical health } \\
\hline Physical function & $\begin{array}{l}\text { Limitations in all (even simple) physical } \\
\text { activities due to health problems }\end{array}$ \\
\hline & Best overall measure of physical health \\
\hline Role physical & $\begin{array}{l}\text { Limitations in work or daily activities } \\
\text { due to physical health }\end{array}$ \\
\hline Bodily pain & Limitations due to severe bodily pain \\
\hline General health & Perceived personal health \\
\hline \multicolumn{2}{|l|}{ Mental health } \\
\hline Vitality & Feelings of vitality, from tired to energetic \\
\hline Social function & $\begin{array}{l}\text { Limitations with social activities due to physical } \\
\text { and emotional problems }\end{array}$ \\
\hline Role emotional & $\begin{array}{l}\text { Problems with work or daily activities } \\
\text { due to emotional problems }\end{array}$ \\
\hline Mental health & $\begin{array}{l}\text { Feelings of depression, nervousness - general } \\
\text { mental health }\end{array}$ \\
\hline & Most valid measure of mental health \\
\hline
\end{tabular}

Note: aHigher scores indicate fewer limitations or problems. 
models with repeated measures (PROC MIXED; SAS Institute Inc) were used to investigate whether the association of physical and mental health component summary scores of the SF-36 with surgical procedure over time differed between treatment groups. An unstructured covariance matrix was specified. Baseline covariates were not included in the models since the uncorrected trajectory of scores over time were of interest, understanding that the two groups were probably different by virtue of the surgery selection process. Residual and Q-Q plots were monitored to check the assumptions of linear modeling. The statistical significance between groups and at different time points was determined using least square mean differences. All $P$-values were corrected for multiple comparisons using the Tukey-Kramer method.

\section{Results}

Thirty-five patients were studied, with 15 patients (42.9\%) undergoing an OSR and 20 patients (57.1\%) undergoing an EVAR. Eleven patients in the OSR group (73.3\%) and 15 patients in the EVAR group (75\%) completed all three follow-up visits. The mean age for all patients was $69.9 \pm 6.5$ years. Only three of the patients $(8.6 \%)$ were women.

Patient baseline clinical characteristics for each group are shown in Table 2. No significant differences in demographic and clinical variables were demonstrated between the OSR and EVAR groups.

Baseline mean SF-36 measurements for the physical and mental health composite scores and subscales (domains) that make up each composite score are shown in Figure 1, along with normative values for visual comparison. ${ }^{17,18}$ There were no statistically significant baseline differences in any of the physical or mental health domain scores between the OSR and the EVAR groups. In the EVAR group, there was a trend to lower mean scores for the domains of PF, RP, and general health. However, none of these differences between study groups were statistically significant. In the repeated measures analysis, baseline physical health composite scores were significantly lower in the EVAR group than in the OSR group $(P<0.05)$, after correction for multiple comparisons.

The SF-36 physical and mental health composite and domain scores over time are demonstrated in Table 3. In the multivariable linear models with repeated measures, both OSR and EVAR groups showed a significant decline in physical health composite scores 30 days after the surgical procedure ( $P<0.01$ and $P<0.01$, respectively). The OSR group showed a statistically significant decline in three of the four physical health domains (PF, $P<0.01$; RP, $P<0.01$; $\mathrm{BP}, P<0.01)$ while the EVAR group declined in only one physical health domain (BP, $P<0.05$ ). Both groups were not significantly different from their baseline by 90 days after the surgery in their physical health composite scores or in any of the respective physical health domains. Regarding mental health composite scores, the OSR group showed a statistically significant decline in three of four mental health domains (VT, $P<0.01$; SF, $P<0.01$; $\mathrm{MH}, P<0.01$ ) at 30 days, but the decline of these domains was not reflected in the OSR group's mental health composite score. Importantly,

Table 2 Demographic, clinical, and baseline variables for all patients, and for patients by surgical procedure

\begin{tabular}{|c|c|c|c|c|}
\hline Variable & Patients $(n=35)$ & OSR group $(n=15)$ & EVAR group $(n=20)$ & $P$-value \\
\hline Age (years) ${ }^{a}$ & $69.9 \pm 6.5$ & $69.2 \pm 3.8$ & $70.5 \pm 8.0$ & 0.74 \\
\hline Male $^{b}$ & 91 (75-98) & $93(66-100)$ & $90(67-98)$ & 1.00 \\
\hline Married ${ }^{b}$ & 77 (59-89) & 87 (59-98) & $70(46-87)$ & 0.60 \\
\hline Smoker ${ }^{\mathrm{b}}$ & $80(63-91)$ & $80(5 I-95)$ & $80(55-93)$ & 1.00 \\
\hline Years smoked ${ }^{a}$ & $29.7 \pm 18.5$ & $28.2 \pm 16.8$ & $30.8 \pm 20.0$ & 0.53 \\
\hline Family history of $A A A^{b}$ & $9(2-25)$ & $7(0-34)$ & $10(2-33)$ & 1.00 \\
\hline Coronary artery disease ${ }^{b}$ & $51(34-68)$ & $47(23-73)$ & $55(32-76)$ & 0.63 \\
\hline Hypertension ${ }^{\mathrm{b}}$ & $54(37-7 I)$ & $53(27-77)$ & $55(32-76)$ & 1.00 \\
\hline Diabetes $^{\mathrm{b}}$ & $14(5-3 \mid)$ & $20(5-49)$ & $10(2-33)$ & 0.63 \\
\hline Cancer $^{\mathrm{b}}$ & $26(13-44)$ & $27(9-55)$ & $25(10-49)$ & 1.00 \\
\hline Cerebral vascular disease ${ }^{\mathrm{b}}$ & $9(2-25)$ & $0(0-27)$ & $15(2-35)$ & 0.25 \\
\hline Previous vascular surgery ${ }^{\mathrm{b}}$ & $3(0-17)$ & $0(0-25)$ & $5(4-27)$ & 1.00 \\
\hline $\mathrm{COPD}^{\mathrm{b}}$ & $20(9-37)$ & $\mid 3(2-4 \mid)$ & $25(10-49)$ & 0.67 \\
\hline $\begin{array}{l}\text { Hysterectomy, prostate, } \\
\text { or testicular surgery }\end{array}$ & $26(13-44)$ & $20(5-49)$ & $30(13-54)$ & 0.70 \\
\hline Bladder repair ${ }^{b}$ & $3(0-17)$ & $0(0-25)$ & $5(0-27)$ & 1.00 \\
\hline
\end{tabular}

Notes: aData presented as mean plus or minus standard deviation; bdata presented as percentage ( $95 \%$ confidence interval).

Abbreviations: OSR, open surgery repair; EVAR, endovascular aneurysm repair; AAA, abdominal aortic aneurysm; COPD, chronic obstructive pulmonary disease. 


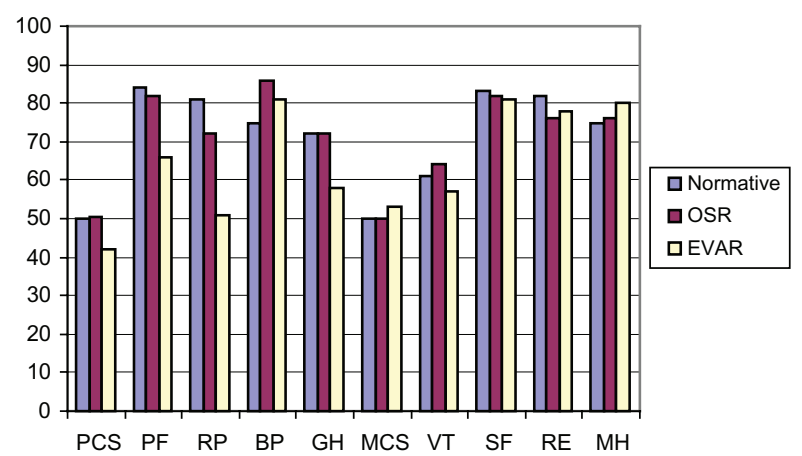

Figure I Baseline physical and mental health composite scores (PCS and MCS, respectively) and their respective domains of the open surgery repair (OSR) and endovascular aneurysm repair (EVAR) groups, as compared with normative scores. Notes: PCS based on four domains: (I) physical function (PF), (2) role physical (RP), (3) bodily pain (BP), (4) and general health (GH); MCS based on four domains: (I) vitality (VT), (2) social function (SF), role emotional (RE), (4) and mental health $(\mathrm{MH})$.

all mental health domain scores returned to baseline values at 90 days, as was seen in the physical health measures.

\section{Discussion}

In clinical trials randomizing patients to elective OSR or EVAR, postoperative outcomes for morbidity and mortality have been similar at 1 year. ${ }^{10,19-21}$ As a disease of the elderly, patients with AAA often have major comorbidities, and
EVAR, as a less invasive procedure, may be the perceived procedure of choice. In contrast, OSR may be preferred if the anatomy of the aneurysm precludes EVAR, as well as in some emergency situations. In other cases the choice of surgical procedure may depend on how the surgeon interprets the current surgical literature, his or her professional experience with the procedures, and, possibly, the patient's perceived physical and mental HRQOL following the surgical technique for AAA repair.

In this study, which represents surgical decision making in a real-world, community hospital setting, it was shown that patients who were selected for EVAR had a trend to lower baseline SF-36 physical health composite scores than those undergoing OSR. This may reflect the clinical decision to prefer EVAR over OSR in patients with poorer health, who may be at a greater risk for complications from open repair.

AAA repair physical health composite scores were substantially reduced 30 days postoperatively for both OSR and EVAR groups. The scores returned to baseline levels for both groups by 90 days, and remained so at 1 year. Neither surgical procedure resulted in changes in the mental health composite scores. These findings were similar to those reported in the randomized EVAR-1 trial, in which physical health composite scores were lower than baseline for the OSR

Table 3 Medical Outcomes Study 36-Item Short-Form Health Survey (SF-36 ${ }^{\circledR}$ ) physical and mental health composite scores and their respective domain scores by procedure and time

\begin{tabular}{|c|c|c|c|c|}
\hline \multirow[t]{2}{*}{ Procedure } & \multicolumn{4}{|c|}{ Follow-up time } \\
\hline & Baseline & 30 days & 90 days & I year \\
\hline \multicolumn{5}{|l|}{ Open surgical repair, baseline SF-36 variable } \\
\hline Overall physical health composite scores & $50.3(7.8)$ & $32.3(7.4)^{\mathrm{a}}$ & $46.9(10.1)$ & $48.9(15.2)$ \\
\hline Physical functioning & $82.7(18.0)$ & $41.9(17.7)^{\mathrm{a}}$ & $77.7(20.6)$ & $86.4(15.2)$ \\
\hline Role physical & $71.7(45.2)$ & $0.0(0.0)^{\mathrm{a}}$ & $50.0(43.9)$ & $72.7(39.5)$ \\
\hline Bodily pain & $85.9(23.9)$ & $49.8(23.0)^{\mathrm{a}}$ & $73.4(26.7)$ & $75.6(19.1)$ \\
\hline General health & $72.0(20.7)$ & $67.2(21.2)$ & $68.5(22.6)$ & $67.5(22.2)$ \\
\hline Overall mental health composite scores & $49.9(9.2)$ & $44.2(11.9)$ & $48.8(9.3)$ & $51.0(10.8)$ \\
\hline Vitality & $64.0(17.1)$ & $33.8(17.8)^{\mathrm{a}}$ & $58.6(19.3)$ & $65.9(21.5)$ \\
\hline Social function & $83.3(21.5)$ & $47.1(21.1)^{\mathrm{a}}$ & $79.5(19.4)$ & $84.1(18.6)$ \\
\hline Role emotional & $75.6(38.8)$ & $51.3(48.3)$ & $52.4(22.7)$ & $81.8(34.5)$ \\
\hline Mental health & $75.8(16.7)$ & $64.6(22.7)^{\mathrm{a}}$ & $75.7(17.5)$ & $75.6(21.4)$ \\
\hline \multicolumn{5}{|l|}{ Endovascular repair, baseline SF-36 variable } \\
\hline Overall physical health composite scores & $42.0(7.5)$ & $34.4(8.2)^{\mathrm{a}}$ & $37.8(10.8)$ & $38.0(11.1)$ \\
\hline Physical functioning & $66.6(16.6)$ & $59.6(24.5)$ & $58.6(28.2)$ & $55.3(28.8)$ \\
\hline Role physical & $5 I .3(4 \mid .7)$ & $18.8(32.3)$ & $44.4(42.5)$ & $46.7(45.2)$ \\
\hline Bodily pain & $80.8(19.5)$ & $56.5(19.1)^{\mathrm{a}}$ & $69.2(29.5)$ & $71.5(25.6)$ \\
\hline General health & $57.7(20.3)$ & $50.3(20.5)$ & $49.2(16.3)$ & $51.3(18.9)$ \\
\hline Overall mental health composite scores & $52.8(8.2)$ & $49.0(10.2)$ & $50.8(9.1)$ & $52.7(8.0)$ \\
\hline Vitality & $56.8(19.8)$ & $47.3(14.7)$ & $46.9(20.6)$ & $52.3(23.6)$ \\
\hline Social function & $80.6(20.1)$ & $65.0(24.2)$ & $72.2(35.0)$ & $80.8(21.6)$ \\
\hline Role emotional & $78.3(34.7)$ & 66.7 (43.9) & 74.1 (38.9) & $77.8(39.2)$ \\
\hline Mental health & 79.5 (12.6) & 7I.I (I2.9) & 76.2 (I3.5) & $74.7(16.5)$ \\
\hline
\end{tabular}

Notes: aStatistically different from baseline, using the Tukey-Kramer method of correction for multiple comparisons. Data presented as mean plus or minus standard deviation. 
group and significantly lower than those for the EVAR group at $0-3$ months, but returned to baseline by 3-12 months. ${ }^{10}$ Analogous to the present study, no changes were observed in the mental health composite scores following OSR or EVAR surgery.

The benefit of administering the validated SF-36 survey is its ability to provide not only psychometrically based physical and mental health composite scores for group comparison but also eight health concepts, or domains, that make up the physical and mental health composite scores for group comparison. ${ }^{16-18}$ This is important, because reporting physical and mental health composite scores alone may miss subtle, yet important, perceived patient-related physical and mental feelings for comparing groups. Those receiving OSR in the present study declined in three (PF, RP, and BP) of the four physical health domains and three (VT, SF, and $\mathrm{MH}$ ) of the four mental health domains at 30 days. In comparison, the EVAR group showed a decrease in only one physical health domain (BP). While both groups experienced reductions in their physical health composite scores, only the OSR group showed a decrease in their PF domain, indicating that the OSR group perceived limitations even in simple, physical activities due to health problems. This finding for the OSR group was not surprising, as these patients had undergone an OSR for AAA just 30 days previously.

Regarding the mental health domains, the OSR group demonstrated declines in VT, SF, and $\mathrm{MH}$, whereas the EVAR group was not found to have any changes in mental health domains at 30 days. These perceived feelings would have remained undetected in the OSR group if only mental health composite scores had been investigated, as these scores showed no change at 30 days postoperatively. Patients in the OSR group perceived themselves as being tired (VT), having physical problems that interfered with social activities (SF), and having feelings of depression and nervousness (MH). Because all physical and mental health domains returned to baseline levels by 90 days in the OSR group, the early advantages of EVAR regarding these domains were only short-lived.

The results of this study utilizing the SF-36 survey demonstrate that patients undergoing either OSR or EVAR have similar decreases in many physical health domains in the early postoperative period. These declines in physical domains for patients undergoing OSR and EVAR were similar, and noted in 4/5 studies for PF, 5/5 studies for RP, and 4/5 studies for BP. Declines for the EVAR group were as follows: 4/5 studies for PF, 5/5 studies for RP, and 4/5 studies for BP. Regarding mental health domains, the OSR groups showed $5 / 5$ studies with a decrease in VT, 4/5 studies with a decrease in SF, and 2/5 studies with a decrease in RE; EVAR groups showed 3/5 studies with a decrease in VT, 4/5 studies with a decrease in SF, and 2/5 studies with a decrease in RE. Interestingly, only the present study demonstrated a decline at 30 days in the $\mathrm{MH}$ domain for the OSR group only.

This suggests that either OSR or EVAR for AAA does not affect patients' perceptions of having poor personal health or patients having a constant feeling of nervousness and depression postoperatively for a life-threatening condition.

Lloyd et $\mathrm{al}^{9}$ reported that both OSR and EVAR groups perceived deterioration in their PF and VT domains at 6 months after surgery. No other earlier time points measuring physical or mental domains were reported in Lloyd et al's ${ }^{9}$ study. In contrast, the present study demonstrated that most domains declined immediately after OSR and EVAR for AAA, but that they returned to baseline values by 3-6 months. As such, the results of the present study are more similar to Prinssen et al's ${ }^{13,19}$ quality-of-life analysis of the Dutch Randomized Endovascular Aneurysm Management trial. An immediate decline in HRQOL after OSR and EVAR, more severe in the former, was followed by recovery approaching baseline levels at an assessment 3 months postoperatively. Interestingly, patients who had undergone OSR seemed to have a higher HRQOL than patients in the EVAR group at the 1 -year assessment. ${ }^{13}$

Most existing data suggest there are early advantages in physical and mental health perception after EVAR, as compared with OSR, although these are of short-term duration. In the present study, for both OSR and EVAR groups, most values were not statistically different from their baseline on any of the physical or mental health composite scores or their domains at 90 days and at 1 year. However, it is possible that some important differences might have been masked by lack of statistical power due to the small sample size.

\section{Conclusion}

In this prospective evaluation of patients undergoing elective AAA repair in a real-world, community hospital setting, the authors demonstrated that patients selected for EVAR suffer less physical and emotional decline in the early postoperative period than those undergoing OSR; however, in both groups a return to near baseline status may occur at 90 days. Information about HRQOL and the specific domains should be presented to patients and considered in addition to traditional clinical parameters when making therapeutic decisions regarding AAA repair. 


\section{Acknowledgments}

The authors would like to acknowledge Bonita Singal, $\mathrm{MD}, \mathrm{PhD}$, for her assistance in the statistical analysis and interpretation.

\section{Disclosure}

NJM and WMW were involved in the study conception and design. NJM, SWL, JVM, RML, and WMW performed the analysis and interpretation. NJM, SWL, and RML collected the data. NJM, SWL, and RML drafted the manuscript and BGH and WMW provided supervision. All authors read and approved the final manuscript.

The authors report no conflicts of interest in this work. No external sources of funding were provided.

\section{References}

1. Harthun NL, Cheanvechal V, Graham LM, Freischlag JA, Gahtan V. Prevalence of abdominal aortic aneurysm and repair outcomes on the basis of patient sex: should the timing of intervention be the same? J Thorac Cardiovasc Surg. 2004;127(2):325-328.

2. National Center for Health Statistics. Deaths, Percent of Total Deaths and Death Rates for the 15 Leading Causes of Death: United States and Each State, 2000. Atlanta, GA: National Vital Statistics System; 2001.

3. Kent KC, Zwolak RM, Jaff MR, et al; Society for Vascular Surgery; American Association of Vascular Surgery; Society for Vascular Medicine and Biology. Screening for abdominal aortic aneurysm: a consensus statement. J Vasc Surg. 2004;39(1):267-269.

4. Brady AR, Fowkes FG, Greenhalgh RM, Powell JT, Ruckley CV, Thompson SG. Risk factors for postoperative death following elective surgical repair of abdominal aortic aneurysm: results from the UK Small Aneurysm Trial. On behalf of the UK Small Aneurysm Trial participants. Br J Surg. 2000;87(6):742-749.

5. Heller JA, Weinberg A, Arons R, et al. Two decades of abdominal aortic aneurysm repair: have we made any progress? J Vasc Surg. 2000;32(6): $1091-1100$

6. Brown MJ, Sutton AJ, Bell PR, Sayers RD. A meta-analysis of 50 years of ruptured abdominal aortic aneurysm repair. Br J Surg. 2002;89(6): 714-730.

7. Hertzer NR. Current status of endovascular repair of infrarenal abdominal aortic aneurysms in the context of 50 years of conventional repair. Ann NY Acad Sci. 2006;1085:175-186.
8. Parodi JC, Palmaz JC, Barone HD. Transfemoral intraluminal graft implantation for abdominal aortic aneurysms. Ann Vasc Surg. 1991;5(6): 491-499.

9. Lloyd AJ, Boyle J, Bell PR, Thompson MM. Comparison of cognitive function and quality of life after endovascular or conventional aortic aneurysm repair. Br J Surg. 2000;87(4):443-447.

10. EVAR trial participants. Endovascular aneurysm repair versus open repair in patients with abdominal aortic aneurysm (EVAR trial 1): randomised controlled trial. Lancet. 2005;365(9478):2179-2186.

11. Aquino RV, Jones MA, Zullo TG, Missig-Carroll N, Makaroun MS. Quality of life assessment in patients undergoing endovascular or conventional AAA repair. $J$ Endovasc Ther. 2001;8(5):521-528.

12. Aljabri B, Al Wahaibi K, Abner D, et al. Patient-reported quality of life after abdominal aortic aneurysm surgery: a prospective comparison of endovascular and open repair. J Vasc Surg. 2006;44(6):1182-1187.

13. Prinssen M, Buskens E, Blankensteijn JD; Dutch Randomized Endovascular Aneurysm Management trial participants. Quality of life endovascular and open AAA repair: results of a randomised trial. Eur J Vasc Endovasc Surg. 2004;27(2):121-127.

14. Lottman PE, Laheij RJ, Cuypers PW, Bender M, Buth J. Healthrelated quality of life outcomes following elective open or endovascular AAA repair: a randomized controlled trial. $J$ Endovasc Ther. 2004;11(3):323-329.

15. Ware JE Jr, Sherbourne CD. The MOS 36-Item Short-Form Health Survey (SF-36): I. Conceptual framework and item selection. Med Care. 1992;30(6):473-483.

16. Ware JE, Snow KK, Kosinski M, Gandek B. SF-36: Health Survey Manual and Interpretation Guide. Boston, MA: The Health Institute, New England Medical Center; 1993.

17. Ware JE Jr, Kosinski M, Keller SK. SF- $36^{\circledR}$ Physical and Mental Health Summary Scales: A User's Manual. Boston, MA: The Health Institute; 1994.

18. Rosenberg W, Donald A. Evidence based medicine: an approach to clinical problem-solving. BMJ. 1995;310(6987):1122-1126.

19. Prinssen M, Verhoeven EL, Buth J, et al; Dutch Randomized Endovascular Aneurysm Management trial group. A randomized trial comparing conventional and endovascular repair of abdominal aortic aneurysms. N Engl J Med. 2004;351(16):1607-1618.

20. Blankensteijn JD, de Jong SE, Prinssen M, et al; Dutch Randomized Endovascular Aneurysm Management trial group. Two-year outcomes after conventional or endovascular repair of abdominal aortic aneurysms. $N$ Engl J Med. 2005;352(23):2398-2405.

21. EVAR trial participants. Endovascular aneurysm repair and outcome in patients unfit for open repair of abdominal aortic aneurysm (EVAR trial 2): randomised controlled trial. Lancet. 2005;365(9478):2187-2192.
Patient Related Outcome Measures

\section{Publish your work in this journal}

Patient Related Outcome Measures is an international, peer-reviewed, open access journal focusing on treatment outcomes specifically relevant to patients. All aspects of patient care are addressed within the journal and practitioners from all disciplines are invited to submit their work as well as healthcare researchers and patient support groups.

\section{Dovepress}

The manuscript management system is completely online and includes a very quick and fair peer-review system. Visit http://www.dovepress. com/testimonials.php to read real quotes from published authors. 